\title{
Inseminación Artificial de Alpacas con Semen Refrigerado y con Inclusión de Dos Tipos de Yema de Huevo
}

\author{
Artificial Insemination of Alpacas with Refrigerated Semen and the Inclusion of \\ Two Types of EgG Yolk
}

Wilber García V. ${ }^{1,3}$, Virgilio Alarcón B. ${ }^{2}$, P. Walter Bravo M. ${ }^{2}$

\section{Resumen}

\begin{abstract}
El objetivo del estudio fue evaluar el efecto de la yema de huevo fresca (YHF) y en polvo (YHP) sobre la motilidad, viabilidad, integridad funcional de la membrana plasmática (HOST) y tasa de preñez con semen fresco y refrigerado. Se colectaron 30 eyaculados de cinco alpacas mediante el método poscópula en los meses de enero a marzo. Los eyaculados fueron diluidos en Tris-glucosa y glicerol a una concentración final de 5\%, y usando los dos tipos de yema de huevo a una concentración final de $20 \%$. El semen diluido se separó en alícuotas y se mantuvo a $15^{\circ} \mathrm{C}$ por $8 \mathrm{~h}$ y a $5{ }^{\circ} \mathrm{C}$ por $24 \mathrm{~h}$, y se inseminaron 180 alpacas. El tipo de yema no afectó la motilidad, viabilidad y el valor HOST en los espermatozoides conservados a 15 y $5^{\circ} \mathrm{C}$. De igual manera, los porcentajes de preñez no variaron cuando se inseminaron con semen fresco $(50.0$ y $46.7 \%)$, mantenido a $15^{\circ} \mathrm{C}(43.3$ y $40 \%)$ o refrigerado a $5^{\circ} \mathrm{C}(33.3$ y $30.0 \%)$ para YHF y YHP, respectivamente. La tasa de preñez fue mayor en hembras inseminadas con semen fresco (48.4\%) y menor con semen refrigerado a $5{ }^{\circ} \mathrm{C}(31.7 \%)(\mathrm{p}<0.05)$. Los resultados sugieren que la yema de huevo en polvo puede utilizarse como substituto de yema de huevo fresco en los diluyentes de conservación y refrigeración de semen de alpacas.
\end{abstract}

Palabras clave: alpaca, inseminación artificial, semen refrigerado, yema de huevo

\section{Abstract}

The aim of this study was to evaluate the effect of fresh (YHF) and powder (YHP) egg yolk on the motility, viability, functional integrity of the plasma membrane (HOST) and pregnancy rate after insemination using fresh and chilled semen. Thirty ejaculates of

\footnotetext{
${ }^{1}$ Centro de Investigación IVITA-Maranganí, Facultad de Medicina Veterinaria, Universidad Nacional Mayor de San Marcos, Lima, Perú

${ }^{2}$ Escuela Profesional de Veterinaria, Universidad Nacional de San Antonio Abad del Cusco (UNSAAC), Cusco, Perú

${ }^{3}$ E-mail: wilberg24@hotmail.com
}

Recibido: 9 de agosto de 2016

Aceptado para publicación: 23 de noviembre de 2016 
five alpacas were collected by the post-copulation method during the months of January to March. Ejaculates were diluted in Tris-glucose plus glycerol to a final concentration of $5 \%$, and the two types of egg yolk were added at a final concentration of $20 \%$. Diluted semen was separated into aliquots and maintained at $15^{\circ} \mathrm{C}$ for $8 \mathrm{~h}$ and $5^{\circ} \mathrm{C}$ for $24 \mathrm{~h}$, where 180 alpacas were inseminated. Motility, viability and sperm HOST value stored at 15 and $5{ }^{\circ} \mathrm{C}$ were not affected by the type of egg yolk. Similarly, pregnancy rates were rather similar when inseminated with fresh semen $(50.0$ and $46.7 \%)$, or kept at $15^{\circ} \mathrm{C}(43.3$ and $40 \%)$ or $5{ }^{\circ} \mathrm{C}(33.3$ and $30.0 \%)$ for YHF and YHP respectively. Pregnancy rate was higher in females inseminated with fresh semen (48.4\%) and lower with chilled semen (31.7\%) $(\mathrm{p}<0.05)$. The results suggested that egg yolk powder can be satisfactorily used as substitute to fresh egg yolk in extenders in fresh and chilled alpaca semen.

Key words: alpaca, artificial insemination, chilled semen, egg yolk

\section{INTRODUCCIÓN}

En varias especies de interés productivo, especialmente en el bovino, la inseminación artificial (IA) constituye la principal herramienta para la diseminación de genes de alta calidad; sin embargo, en camélidos sudamericanos (CSA) se limita a la utilización de semen fresco con una tasa máxima de preñez del 77\% (alpacas: Alarcón et al., 2012; llamas: Giuliano et al., 2012).

La IA con semen refrigerado en CSA presenta resultados muy variables. Así Giuliano et al. (2012) reportan 13\% de tasa de preñez en llamas y Bravo y Alarcón (2013) señalan $58 \%$ en de preñez en alpacas. Una de las posibles causas de esta variabilidad podría estar en la utilización de yema de huevo fresco como parte del diluyente en la conservación del semen (Bravo y Alarcón, 2013), por sus propiedades para estabilizar la membrana del espermatozoide (Salamon y Maxwell, 2000). Sin embargo, la heterogeneidad de la composición entre lotes de huevos, junto con el potencial riesgo de contaminación microbiológica, conlleva a la posibilidad de utilizar un sustituto. La yema de huevo en polvo podría ser una alternativa, ya que este producto se somete a un proceso de pasteurización para destruir las bacterias, de acuerdo con las leyes establecidas para el consumo humano (Thibier y Guerin, 2000) y los lotes tienen una composición homogénea. Es así, que el presente trabajo evaluó el efecto de dos tipos de yema de huevo (fresca y en polvo) sobre la motilidad, viabilidad, HOST y tasa de preñez de los espermatozoides pre y pos-refrigerados.

\section{Materiales y Métodos}

\section{Lugar de Estudio y Animales}

El estudio se realizó en el Centro Experimental de Camélidos Sudamericanos La Raya, de la Universidad Nacional San Antonio Abad, situado en el distrito de Maranganí, Cusco, Perú, a una altitud de $4200 \mathrm{msnm}$. Se utilizaron cinco alpacas machos, de 4 a 8 años de edad y peso promedio de $60 \pm 6.4 \mathrm{~kg}$, así como 180 alpacas hembras, de 3 a 7 años de edad y peso promedio de $55 \pm 7.2 \mathrm{~kg}$. Los animales se mantuvieron en pasturas naturales durante todo el estudio. Asimismo, los machos eran parte del grupo élite de reproductores del centro.

\section{Colección y Refrigeración de Semen}

La colección se realizó entre los meses de enero a marzo. Las muestras de semen fueron obtenidas poscópula, de acuerdo con la técnica descrita por Neely y Bravo (1998), 
para lo cual se utilizaron hembras adultas sexualmente receptivas al macho. La colección de semen se realizó dos veces por semana.

Cada hembra fue empadrada con un macho. Al término de la cópula se insertó un espéculo vaginal y todo el semen que se encontraba en la vecindad externa de la cérvix fue colectado y depositado en un tubo de vidrio graduado y mantenido a $37^{\circ} \mathrm{C}$. Se evaluó la calidad del semen fresco, aceptando los eyaculados que cumplieron los siguientes requisitos: volumen $\geq 1 \mathrm{ml}$ y motilidad total $\geq 60 \%$. Se trabajó con 30 eyaculados (6 eyaculados por macho).

Las muestras de semen fueron divididas en seis alícuotas, las cuales se diluyeron con una solución compuesta por $0.3 \mathrm{M}$ Tris, $27.75 \mathrm{mM}$ glucosa, $94.7 \mathrm{mM}$ ácido cítrico, ajustada a un $\mathrm{pH} 7.25 \pm 0.05$ y una osmolaridad de $333.0 \pm 2.80 \mathrm{mOsm}$. A esta solución se le añadió tilosina $0.1 \mathrm{mg} / \mathrm{ml}$, gentamicina $0.5 \mathrm{mg} / \mathrm{ml}$ y lincomicina $1.8 \mathrm{mg}$ $\mathrm{mg} / \mathrm{ml}$ y uno de los dos tipos de yema de huevo: yema de huevo fresco (YHF) o yema de huevo en polvo (YHP) a una concentración final de $20 \%$ (v/v). Las alícuotas de esta forma fueron mantenidas a $35^{\circ} \mathrm{C}$, y luego a 15 ${ }^{\circ} \mathrm{C}$ por $8 \mathrm{~h}$ y refrigeradas a $5^{\circ} \mathrm{C}$ por $24 \mathrm{~h}$.

\section{Variables de Calidad Seminal}

El volumen de cada eyaculado fue determinado en un tubo graduado. Para la motilidad espermática, se tomó $10 \mu \mathrm{l}$ de semen en una lámina portaobjetos precalentada, cubriéndola con una laminilla y observada a 400X en un microscopio de contraste de fase. La motilidad se calificó como porcentaje de espermatozoides con movimientos oscilatorios y progresivos en un campo microscópico como lo describe Garnica et al. (1993). La motilidad se expresó en porcentaje.

La viabilidad espermática en fresco y tras el enfriamiento a 15 y $5^{\circ} \mathrm{C}$ se evaluó por medio de la tinción de eosina-nigrosina
(Hancok, 1957), en portaobjetos atemperados a $35{ }^{\circ} \mathrm{C}$ y con la ayuda de un microscopio óptico a 400X. Se contaron 200 espermatozoides en varios campos del microscopio. Se consideraron como espermatozoides vivos aquellos donde el colorante no penetró en la cabeza. La viabilidad se expresó en porcentaje.

La concentración espermática se determinó en una cámara de Newbauer. Una muestra de semen se diluyó 1:50 o 1:100, de acuerdo a la evaluación previa de la motilidad, en solución de $\mathrm{NaCl}$ al 3\%. La concentración espermática se expresó en millones de espermatozoides por mililitro.

El test de endosmosis (Hypoos-moticSwelling test, HOST) se hizo con la finalidad de evaluar la integridad funcional de la membrana plasmática. Para esto, se incubaron $25 \mu \mathrm{L}$ de la muestra en $500 \mu \mathrm{L}$ de solución hipoosmótica (100 mOsm) durante 60 min a $37^{\circ} \mathrm{C}$ y luego se observó $10 \mu \mathrm{L}$ de la muestra al microscopio. Un total de 200 espermatozoides fueron contados en varios campos del microscopio, donde los espermatozoides vivos con colas dobladas e hinchadas fueron registrados como células positivas a HOST (Aisen, 2004). Los resultados se expresaron en porcentaje.

\section{Inseminación Artificial}

Se seleccionaron 180 alpacas adultas con historia de partos previos y con un descanso sexual mínimo de 20 días posparto. Los animales que presentaban conducta manifiesta de recepción del macho y un folículo preovulatorio $\geq 7 \mathrm{~mm}$ detectado por ecografía transrectal, mediante un ecógrafo Aloka 500 (Aloka Co., Led Tokio, Japón) equipado con un transductor lineal de $7.5 \mathrm{MHz}$, fueron inducidas a ovular con la administración intramuscular de $80 \mu \mathrm{g}$ buserelina por animal (Bravo et al., 2012).

Las alpacas fueron inseminadas a las 26-30 h (Gonzáles et al., 2011) de la inducción de la ovulación. La región vulvar fue lim- 
Cuadro 1. Motilidad (\%), viabilidad (\%) y HOST (\%) de los espermatozoides conservados en fresco, mantenidos a $15^{\circ} \mathrm{C}$ por 8 horas y refrigerados a $5{ }^{\circ} \mathrm{C}$ por 24 horas en yema de huevo fresco (YHF) y yema de huevo en polvo (YHP)

\begin{tabular}{|c|c|c|c|c|}
\hline \multirow{2}{*}{ Variables } & \multirow{2}{*}{ Diluyente } & \multicolumn{3}{|c|}{ Temperatura $\left({ }^{\circ} \mathrm{C}\right)$} \\
\hline & & Fresco & 15 & 5 \\
\hline \multirow{2}{*}{ Motilidad } & YHF & \multirow{2}{*}{$61.7 \pm 2.3^{\mathrm{a}}$} & $55.3 \pm 2.0^{\mathrm{ab}}$ & $48.9 \pm 0.5^{b}$ \\
\hline & YHP & & $50.9 \pm 1.1^{b}$ & $45.9 \pm 1.4^{\mathrm{b}}$ \\
\hline \multirow{2}{*}{ Viabilidad } & YHF & \multirow{2}{*}{$71.6 \pm 2.2^{\mathrm{a}}$} & $64.3 \pm 2.0^{\mathrm{b}}$ & $58.3 \pm 3.1^{\mathrm{b}}$ \\
\hline & YHP & & $67.1 \pm 2.1^{\mathrm{ab}}$ & $60.3 \pm 3.3^{b}$ \\
\hline \multirow{2}{*}{ HOST } & YHF & \multirow{2}{*}{$52.0 \pm 2.4^{\mathrm{a}}$} & $45.3 \pm 2.8^{\mathrm{ab}}$ & $39.8 \pm 1.4^{\mathrm{b}}$ \\
\hline & YHP & & $46.5 \pm 2.3^{\mathrm{ab}}$ & $41.4 \pm 3.4^{\mathrm{b}}$ \\
\hline
\end{tabular}

${ }^{a b}$ Superíndices diferentes dentro de filas indican diferencia significativa $(p<0.05)$

Cuadro 2. Porcentaje de preñez a los 30 días pos-inseminación de alpacas $(\mathrm{n}=30$ por tratamiento) con espermatozoides conservados en fresco $\left(35^{\circ} \mathrm{C}\right)$, a $15^{\circ} \mathrm{C}$ por 8 horas y refrigerado a $5{ }^{\circ} \mathrm{C}$ por 24 horas utilizando yema de huevo fresco (YHF) o yema de huevo en polvo (YHP) como componente del dilutor

\begin{tabular}{ccc}
\hline Tratamiento & Temperatura $\left({ }^{\circ} \mathrm{C}\right)$ & Tasa de preñez $(\%)$ \\
\hline YHF & 35 & $50.0^{\mathrm{a}}$ \\
YHP & 35 & $46.7^{\mathrm{a}}$ \\
YHF & 15 & $43.3^{\mathrm{ab}}$ \\
YHP & 15 & $40.0^{\mathrm{ab}}$ \\
YHF & 5 & $33.3^{\mathrm{b}}$ \\
YHP & 5 & $30.0^{\mathrm{b}}$ \\
\hline
\end{tabular}

${ }^{\mathrm{ab}}$ Superíndices diferentes dentro de columna indican diferencia significativa $(p<0.05)$

piada con una toalla húmeda y se introdujo el espéculo vaginal para identificar la os externa de la cérvix. Se utilizó semen fresco diluido a $35^{\circ} \mathrm{C}$ por $10 \mathrm{~min}$, enfriado a $15^{\circ} \mathrm{C}$ por $8 \mathrm{~h}$ y refrigerado a $5^{\circ} \mathrm{C}$ por $24 \mathrm{~h}$. El semen diluido $(1 \mathrm{ml})$ a una concentración de $25 \times 10^{6} / \mathrm{ml}$ fue cargado en una pipeta de inseminación de vacunos y se introdujo dentro del cuerpo del útero. El diagnóstico de preñez se hizo por ecografía a los 30 días del servicio. La presencia de la vesícula embrionaria dentro del útero fue considerada como preñez. 


\section{Análisis Estadístico}

Se empleó estadística descriptiva para determinar el tiempo de cópula, volumen del eyaculado y concentración. Los porcentajes de motilidad, viabilidad y HOST + fueron transformados a valores angulares (ángulo=arcoseno Vporcentaje final) para acercar los datos a la distribución normal. Para determinar la existencia de diferencias estadísticas se utilizó la prueba de análisis de varianza, así como la prueba de Duncan para determinar diferencias estadísticas entre grupos. Para evaluar el porcentaje de preñez, se empleó una regresión logística con el procedimiento Genmod del SAS 9.1.

\section{Resultados}

El tiempo promedio de cópula, volumen y concentración espermática de los 30 eyaculados colectados fue de $19.5 \pm 5.7 \mathrm{~min}$, $2.9 \pm 0.6 \mathrm{ml}$ y $76.2 \pm 15.2 \times 10^{6} / \mathrm{ml}$, respectivamente.

Los valores de motilidad, viabilidad y HOST (Cuadro 1) de los espermatozoides conservados en refrigeración a $5{ }^{\circ} \mathrm{C}$ disminuyeron significativamente $(p<0.05)$ en comparación con las muestras en fresco en los dos tipos de yema de huevo. En cambio, los espermatozoides conservados en fresco proporcionaron valores superiores de HOST, aunque no significativos, con los observados a los mantenidos a $15^{\circ} \mathrm{C}$ en los dos tipos de yema de huevo. En general, no se encontró diferencia significativa por efecto del tipo de yema de huevo en la motilidad, viabilidad y HOST en los espermatozoides mantenidos a $15^{\circ} \mathrm{C}$ y refrigerados.

Los resultados de la tasa de preñez se presentan en el Cuadro 2. La mayor tasa de preñez se presentó en hembras inseminadas con semen fresco $(48.4 \%)$ y la menor tasa de preñez con semen refrigerado a $5{ }^{\circ} \mathrm{C}$ $(31.7 \%)(p<0.05)$. Por otro lado, no hubo diferencia significativa en preñez por efecto del tipo de yema de huevo en las tres temperaturas en estudio.

\section{Discusión}

Diversos investigadores han demostrado que la yema de huevo en los diluyentes de preservación protegen a los espermatozoides de los mamíferos del shock por frío, y preservan la integridad de las membranas plasmática, acrosomal y mitocondrial, manteniendo la motilidad y fertilidad de los espermatozoides de toro (Wall y Foote, 1999), carnero (Salamon y Maxwell 1995; Ali et al., 2013), macho cabrío (Cabrera et al., 2005), alpaca (Bravo et al., 2000), llama (Bravo et al., 2013) y camello (Deen et al., 2003). Sin embargo, la yema de huevo, a pesar de ser el protector no penetrante más comúnmente usado por su capacidad de estabilizar la membrana del espermatozoide (Salamon y Maxwell, 2000), contiene sustancias que pueden interferir en el metabolismo celular reduciendo la motilidad espermática (Moussa et al., 2002), además de la dificultad de obtener lotes uniformes de huevos en algunas zonas del país.

Los resultados del presente estudio indican pequeñas diferencias en el uso de la yema de huevo fresca y en polvo en lo que respecta a la protección de los espermatozoides durante el proceso de conservación a $15^{\circ} \mathrm{C}$ y refrigeración. No se apreció un efecto marcado del tipo de yema de huevo en términos de motilidad, viabilidad, integridad de la membrana plasmática y fertilidad de las alpacas inseminadas con espermatozoides en fresco, conservados a $15^{\circ} \mathrm{C}$ o refrigerados. Resultados similares han sido reportados por Marco-Jiménez et al. (2004) al comparar yema de huevo en polvo con yema de huevo fresco en ovinos de la raza Guirra respecto a la integridad del acrosoma, aunque sí detectaron un incremento significativo en la motilidad total en semen refrigerado a favor de la yema de huevo en polvo. Wall y Foote (1999) tampoco encontraron diferencias en viabilidad y fertilidad espermática de semen refrigerado de toro al comparar yema de huevo clarificada con yema de huevo fresco, mientras que Fernández-Santos et al. (2006) 
observaron una mejora en la calidad espermática con el uso de yema de huevo clarificado en la refrigeración de espermatozoides del ciervo Ibérico. Las diferencias en resultados entre especies pueden deberse a las diferencias en la composición de membrana plasmática, en el porcentaje de yema de huevo en el diluyente, en los aditivos incluidos y en el tiempo de refrigeración (Fernández-Santos et al., 2006; Waterhouse et al., 2006).

Los resultados de motilidad por efecto de la temperatura y tiempo durante la refrigeración fueron similares al estudio de Morton et al. (2010) con espermatozoides de epidídimo de alpaca utilizando Biladyl ${ }^{\circledR}$ como diluyente. Asimismo, los valores de viabilidad y HOST fueron similares an semen refrigerado de llama (Giuliano et al., 2012), alpaca (Bravo y Alarcón, 2013) y ovino (Yániz et al., 2011), mientras que Vaughan et al. (2003) en espermatozoides de alpaca, obtuvieron porcentajes inferiores en diluyentes comerciales sin productos de origen animal (Andromed ${ }^{\circledR}$; Biociphos ${ }^{\circledR}$; Plus ${ }^{\circledR}$ y Bioxcell $\left.{ }^{\circledR}\right)$.

El 48\% de tasa de preñez obtenido con semen fresco fue significativamente superior $(\mathrm{p}<0.05)$ al obtenido con semen refrigerado a $5{ }^{\circ} \mathrm{C}(37 \%)$. Esta diferencia puede atribuirse al cambio de temperatura de la suspensión espermática que se reduce desde valores fisiológicos hasta los $5{ }^{\circ} \mathrm{C}$, poniendo de manifiesto la extrema sensibilidad de los espermatozoides de alpaca al descenso de temperatura, más conocido como «shock por frío» (Parks, 1997). El daño sufrido durante este proceso afecta principalmente a las membranas, observándose alteraciones morfológicas, tanto en la membrana plasmática como en la acrosomal, pero, además, también se producen cambios bioquímicos a otros niveles, como la disminución de la actividad respiratoria y daño en el DNA (Weitze y Petrunkina, 2007). Este proceso ha sido descrito en los espermatozoides de cerdo (Watson, 1996), toro (Cormier et al., 1997), carnero (Gillan et al., 1997), potro (Thomas et al., 2006) y alpaca (Canorio, 2008).

\section{Conclusiones}

Los resultados sugieren que la yema de huevo en polvo puede utilizarse en forma satisfactoria en los diluyentes para la inseminación artificial de alpacas, tanto como semen fresco, conservado a $15^{\circ} \mathrm{C}$ por 8 horas y refrigerado a $5^{\circ} \mathrm{C}$ por 24 horas, sustituyendo a la yema de huevo fresco.

\section{Agradecimiento}

Los autores agradecen al Consejo Superior de Investigaciones (CSI) de la Universidad Nacional Mayor de San Marcos por el financiamiento otorgado para el desarrollo del estudio.

\section{Literatura Citada}

1. Aisen E. 2004. Reproducción ovina y caprina. Argentina: Inter-Medica. 205 p.

2. Alarcón V, García W, Bravo, W. 2012. Inseminación artificial de alpacas con semen colectado por aspiración vaginal y vagina artificial. Rev Inv Vet Perú 23: 58-64. doi: 10.15381/rivep.v23i1.882

3. Ali A, Bomboi G, Floris B. 2013. Replacing chicken yolk with yolks from other sources in ram semen diluents and their effects on fertility in vitro. Small Rum Res 113: 405-410. doi: 10.1016/ j.smallrumres.2013.01.017

4. Bravo W, Skidmore A, Zhao X. 2000. Reproductive aspects and storage of semen in Camelidae. Anim Reprod Sci 62: 173-193. doi: 10.1016/S03784320(00)00158-5

5. Bravo W, Alarcón V, García W. 2012. New developments on artificial insemination of llamas and alpacas. Proc ICAR 2102 Satellite Meeting on Camelid Reproduction. Vancouver, Canada. 
6. Bravo W, Alarcón V. 2013. Preservación de semen y avances recientes en la inseminación artificial de llamas y alpacas. Spermova 3: 158-160.

7. Bravo W, Alarcón V, Baca L, Cuba Y, Ordoñez C, Salinas J, Tito F. 2013. Semen preservation and artificial insemination in domesticated South American camelids. Anim Reprod Sci 136: 157- 163. doi: 10.1016/j.anireprosci.2012.10.005

8. Cabrera F, González F, Batista M, Calero P, Medrano A, Gracia A. 2005. The effect of removal of seminal plasma, egg yolk level and season on sperm freezability of canary buck (Capra hircus). Reprod Dom Anim 40: 191-195. doi: 10.1111/j.1439-0531.2005.00544.x

9. Canorio N. 2008. Criocapacitación del espermatozoide de alpaca (Lama pacos). Tesis de Magister. Lima: Univ Nacional Mayor de San Marcos. 73 p.

10. Cormier N, Sirard M, Bailey J. 1997. Premature capacitation of bovine spermatozoa is initiated by cryopreservation. J Androl 18: 461-468.

11. Deen A, Vyas S, Sahani M. 2003. Semen collection, cryopreservation and artificial insemination in the dromedary camel. Anim Reprod Sci 77: 223-233. doi: 10.1016/S0378-4320(03)00040-X

12. Fernández-Santos $R$, Esteso $C$, Montoro, V, Soler J, Garde J. 2006. Cryopreservation of Iberian red deer (Cervus elaphus hispanicus) epididymal spermatozoa: effects of egg yolk, glycerol and cooling rate. Theriogenology 66: 1931-1942. doi: 10.1016/j.theriogenology.2006.05.012

13. Garnica J, Achata $R$, Bravo $P W$. 1993. Physical and biochemical characteristics of alpaca semen. Anim Reprod Sci 32: 85-90. doi: 10.1016/03784320(93)90059-Z

14. Gillan L, Evans G, Maxwell W. 1997. Capacitation status and fertility of fresh and frozen-thawed ram spermatozoa. Reprod Fertil Dev 9: 481-487.
15. Giuliano S, Chavez M, Trasorras V, Gambarotta M, Neild D, Director A, Pinto M, Miragaya M. 2012. Development of an artificial insemination protocol in llamas using cooled semen. Anim Reprod Sci 131: 204-210. doi: 10.1016/j.anireprosci.2012.03.010

16. Gonzáles M, Huanca T, Cárdenas $O$. 2011. Evaluación de la fertilidad en alpacas inseminadas con semen refrigerado a diferentes tiempos post inducción de ovulación. Spermova 1: 102-103.

17. Hancock J. 1957. The morphology of boar spermatozoa. JR Microsc Soc 76: 84-97.

18. Marco-Jiménez F, Puchades S, Mocé $E$, Viudes-De-Cartro M, Vicente J, Rodriguez M. 2004. Use of powdered egg yolk vs fresh egg yolk for the cryopreservation of ovine semen. Reprod Dom Anim 39: 438-441. doi: 10.1111/j.1439-0531.2004.00537.x

19. Morton K, Evans G, Maxwell W. 2010. Effect of glycerol concentration, Equex STM ${ }^{\circledR}$ supplementation and liquid storage prior to freezing on the motility and acrosome integrity of frozen-thawed epididymal alpaca (Vicugna pacos) sperm. Theriogenology 74: 311-316. doi: 10.1016/j.theriogenology.2010.02.015

20. Moussa M, Martinet V, Trimeche A, Tainturier D, Anton M. 2002. Low density lipoproteins extracted from hen egg yolk by an easy method: cryoprotective effect on frozen-thawed bull semen. Theriogenology 57: 16951706. doi: 10.1016/S0093-691X(02)00682-9

21. Neely DP, Bravo PW. 1998. Reproductive evaluation and infertility in the male llama and alpaca. In: Youngquist RS (ed). Current therapy in large animal theriogenology. USA: WB Saunders. p 787-792.

22. Parks J. 1997. Hypothermian and mammalian gametes. In: Karow AM, Critser JK (eds). Reproductive tissue banking: scientific principles. San Diego, EEUU: Academic Press. p 229-261. 
23. Salamon S, Maxwell W. 1995. Frozen storage of ram semen II. Causes of low fertility after cervical insemination and methods of improvement. Anim Reprod Sci 38: 1-36. doi: 10.1016/0378-4320 (94)01328-J

24. Salamon S, Maxwell W. 2000. Storage of ram semen. Anim Reprod Sci 62: 77111. doi: 10.1016/S0378-4320(00)00155-X

25. Thibier M, Guerin B. 2000. Hygienic aspects of storage and use of semen for artificial insemination. Anim Reprod Sci 62:233-251.

26. Thomas A, Meyers S, Ball A. 2006. Capacitation-like changes in equine spermatozoa following cryopreservation. Theriogenology 65: 1531-1550. doi: 10.1016/S0378-4320(00)00161-5

27. Wall J, Foote H. 1999. Fertility of bull sperm frozen and stored in clarified egg yolk-Tris-glycerol extender. J Dairy Sci 82: 817-821. doi: $10.3168 /$ jds.S00220302(99)75301-4

28. Waterhouse E, Hofmo O, Tverdal A, Miller R. 2006. Within and between breed differences in freezing tolerance and plasma membrane fatty acid composition of boar sperm. Reproduction 131: 887-894. doi: 10.1530/rep.1.01049

29. Watson P. 1996. Cooling of spermatozoa and fertilizing capacity. Reprod Dom Anim 31: 135-140. doi: 10.1111/j.14390531.1995.tb00016.x

30. Weitze K, Petrunkina A. 2007. Conservación del semen. Bases bioquímicas y principios de las técnicas de congelación y descongelación. En: Busch W, Waberski D (eds). Manual de inseminación artificial de los animales domésticos y de explotación zootécnica. España: Acribia. p 139-153.

31. Vaughan J, Galloway D, Hopkins D. 2003. Artificial insemination in alpacas (Lama pacos). RIRDC Rural Industries Research and Development Corporation, Pub. No. 03/104, Kingston, Australia. p 74-77.

32. Yániz L, Mateos A, Santolaria P. 2011. Zwitterionic buffers preserve ram semen quality more efficiently than TRIS during storage at $15^{\circ} \mathrm{C}$. Small Rum Res 95: 5460. doi: 10.1016/j.smallrum-res.2010.08.006 